\title{
Inhibition of Synovial Macrophage Pyroptosis Alleviates Synovitis and Fibrosis in Knee Osteoarthritis
}

\author{
Li Zhang, ${ }^{1}$ Runlin Xing $\mathbb{D},{ }^{1}$ Zhengquan Huang, ${ }^{1}$ Nongshan Zhang, ${ }^{1}$ Li Zhang, ${ }^{1}$ Xiaochen Li, \\ and Peimin Wang $\mathbb{D}^{1,2}$ \\ ${ }^{1}$ The Affiliated Hospital of Nanjing University of Chinese Medicine, Department of Orthopedics, Nanjing, China \\ ${ }^{2}$ Hospital of Nanjing University of Chinese Medicine, No. 155 Hanzhong Road, Nanjing, Jiangsu Province, China \\ Correspondence should be addressed to Peimin Wang; drwpm@163.com
}

Received 16 May 2019; Accepted 11 August 2019; Published 8 September 2019

Academic Editor: Maria Jose Alcaraz

Copyright ( 2019 Li Zhang et al. This is an open access article distributed under the Creative Commons Attribution License, which permits unrestricted use, distribution, and reproduction in any medium, provided the original work is properly cited.

\begin{abstract}
Increasing evidence has shown that macrophage pyroptosis in different tissues participates in chronic aseptic inflammation and is related to tissue fibrosis. Our last studies also revealed the vital role of synovial fibroblast pyroptosis in the onset and development of knee osteoarthritis (KOA). In this study, we aimed to investigate whether synovial macrophage pyroptosis did occur and whether this form of cell death should be related to synovitis and fibrosis of KOA. In the synovial tissue of KOA model rats, we observed a decrease of caspase1, NLRP3, ASC, and GSDMD caused by macrophage depletion in both the mRNA and protein expressions. Besides, rats treated with the specific caspase1 inhibitor Ac-YVAD-CMK showed less inflammatory reaction and fibrosis, not only in the expression of proinflammatory factors IL-1 $\beta$, IL-18, and HMGB1 and fibrosis markers TGF- $\beta$, PLOD2, COL1A1, and TIMP1 but also in the observation of HE staining, Sirius Red staining, and the transverse diameters of the right knees. Subsequently, we established an LPS+ATP-induced model in macrophages mimicking the inflammatory environment of KOA and inducing macrophage pyroptosis. Macrophages transfected with caspase1 siRNA showed reduced cell death; meanwhile, the relative expression of pyroptosis-related proteins were also downregulated. In addition, the level of fibrotic markers in synovial fibroblasts were significantly decreased after coculture with siRNA GSDMD-transfected macrophages. To conclude, synovial macrophage pyroptosis may occur in the pathological processes of KOA and inhibition of synovial macrophage pyroptosis alleviates synovitis and fibrosis in KOA model rats.
\end{abstract}

\section{Introduction}

Knee osteoarthritis (KOA) is a degenerative joint disorder that affects all tissues in the knees, while chronic lowgrade inflammation is a major driver of the ongoing joint degeneration [1]. Symptomatically, KOA is characterized by the progressive destruction of articular cartilage and surrounding tissues, especially synovial tissue, causing pain, stiffness, and disability [2]. Synovial tissue is the soft membrane lining the spaces of knees and contains highly metabolically active cells called synoviocytes which are further separated as synovial macrophages and synovial fibroblasts. These cell types and the synovial tissue as a whole play a key role in normal joint physiology as they facilitate nourishment for chondrocytes and remove the waste products through a synovial fluid [3]. So far, we lack a compre- hensive understanding of the pathogenesis of KOA, and the present study generally agrees that synovitis plays an important role in the development of KOA.

The infiltration and activation of macrophages in synovial tissue make synovitis a primary trigger of KOA's pathogenesis. Its importance has been implicated by their close relevance to KOA symptoms such as cartilage destruction and synovial fibrosis [4]. The fact that macrophages are involved in KOA even makes them valid candidates to use in the quantitative assessment of inflammation [5]. Synovial fibrosis is another pathological change of synovial tissue characterized by excessive extracellular matrix deposition, which contributes to joint pain and stiffness. Genes procollagen-lysine, 2-oxoglutarate 5-dioxygenase 2 (PLOD2), collagen type I $\alpha 1$ chain (COL1A1), and tissue inhibitor of metalloproteinase 1 (TIMP1) are shown upregulating in 
osteoarthritis-related fibrosis; they are usually considered to be fibrotic markers [6]. Besides, accumulating evidence has demonstrated the critical role of synovial fibroblasts and transforming growth factor- $\beta$ (TGF- $\beta$ ) in fibrotic response $[7,8]$.

Recently, a new form of programmed cell death, mostly found in monocytes, macrophages, and dendritic cells, has been described as pyroptosis [9]. In the canonical pathway of pyroptosis, NLRP3, the most well-studied nod-like receptor, forms an inflammasome comprised of apoptosisassociated speck-like protein (ASC) and the serine protease caspase1 [10]. NLRP3 inflammasome activation governs the cleavage and activation of pro-caspase1. Finally, activated caspase 1 causes proinflammatory cytokines such as pro-IL$1 \beta$, pro-IL-18, and HMGB1 to mature and induces pyroptosis depending on the cleavage of gasdermin D (GSDMD) $[11,12]$. In our last studies, the correlation between the NLRP inflammasomes and synovial fibroblast pyroptosis was investigated in vivo and in vitro [13]. We also revealed that increased hypoxia-inducible factor- $1 \alpha$ in KOA aggravates synovial fibrosis via synovial fibroblast pyroptosis [14].

A study on macrophage pyroptosis is initially concentrated on infection caused by bacteria and viruses [15]. But increasing evidence has shown that macrophage pyroptosis in different tissues participates in chronic aseptic inflammation and is related to tissue fibrosis. For instance, microglia (i.e., macrophages of the central nervous system) pyroptosis can induce neurogenic inflammation and fibrosis [16], liver Kupffer cell (i.e., macrophages in liver tissue) pyroptosis can cause chronic hepatitis and liver fibrosis [17], and macrophage pyroptosis in the kidney can lead to renal inflammation and fibrosis [18].

In summary, KOA shows a state of low-grade inflammation and a combination of synovitis and fibrosis. These pathological processes are remarkably similar to those "macrophage pyroptosis/inflammation/fibrosis" in the field of other tissues. Accordingly, we hypothesized that there should be macrophage pyroptosis in synovial tissues and this form of cell death should be related to synovitis and fibrosis.

\section{Materials and Methods}

2.1. In Vivo Animal Experimental Design. Thirty-two 3month-old SD male rats, with weights ranging from $250 \mathrm{~g}$ to $290 \mathrm{~g}$ (provided by Beijing Vital River Laboratory Animal Technology Co. Ltd.), were used. Animals were maintained in a specific pathogen-free, laminar-flow housing apparatus under controlled temperature, humidity, and $12 \mathrm{~h}$ light/dark regimen. All animal protocols were approved by the Animal Care and Use Committee of the Nanjing University of Chinese Medicine. All experiments were conducted in accordance with the National Institutes of Health Guidelines for the Care and Use of Laboratory Animals.

Rats were randomly assigned to the following four groups (with eight rats in each group): Normal, KOA, KOA+Clodronate Liposomes $(\mathrm{KOA}+\mathrm{CL})$, and $\mathrm{KOA}+\mathrm{Ac}-\mathrm{YVAD}-\mathrm{CMK}$ (KOA+AYC). On Day 1, the KOA model was constructed by anterior cruciate ligament transection (ACLT) surgery in both knees as described previously [19]. 14 days after surgery
(Day 14), drug administration began. The KOA+CL group was depleted of synovial lining macrophages by intraarticular injection of $100 \mu \mathrm{l}$ Clodronate Liposomes (Clodronate Liposomes, Amsterdam, Netherlands) [20]. The $\mathrm{KOA}+\mathrm{AYC}$ group was intra-articularly injected with caspase1 inhibitor Ac-YVAD-CMK (Sigma-Aldrich, St. Louis, $\mathrm{MO}, \mathrm{USA}$ ) at a dose of $0.25 \mathrm{mg} / \mathrm{kg}$ (in saline with $10 \%$ DMSO, $100 \mu \mathrm{l})$. The Normal group and the KOA group were treated with $100 \mu \mathrm{l}$ sterilized physiologic saline at the same time. All drugs were injected every 2 days for 2 weeks. After that, rats were sacrificed to harvest the synovial tissue.

2.2. Measurement of the Right Knee Joint Diameter. The transverse diameters of the right knees were measured with a slide caliper at Day 1, Day 7, Day 14, Day 21, and Day 28 (Mitutoyo, Kanagawa, Japan) to measure the horizontal distance between the left and right highest points of the knee joints flexed at $90^{\circ}$, respectively. Each knee was measured three times, and the mean value was calculated.

2.3. Immunofluorescence Assay. The synovial tissues were frozen for the preparation of sections. Sections were washed in $\mathrm{PBS}$ for $5 \mathrm{~min}$ and processed in $0.3 \% \mathrm{H}_{2} \mathrm{O}_{2}$ in methanol for 20 minutes. The goat serum was added to the block for $30 \mathrm{~min}$ at room temperature. Then, the sections were incubated with primary antibodies of anti-CD68 (a panmacrophage marker [21], Abcam, Cambridge, UK) at $4^{\circ} \mathrm{C}$ overnight and with secondary antibodies of anti-goat/rabbit at $37^{\circ} \mathrm{C}$ for $2 \mathrm{~h}$. CD68 was stained with bright orange fluorescence, and sections were observed under a fluorescence microscope (Zeiss, Germany).

2.4. Histological Analysis. Synovial tissues were collected after executing rats, and tissues were then fixed in $10 \%$ neutral formalin, embedded in paraffin, and cut into slices for routine HE staining. Sirius Red staining was carried out according to the instructions of the Sirius Red Stain Kit (Beyotime Biotechnology, Shanghai, China). Briefly, the tissue sections were dewaxed, dipped into water, stained with $1 \mathrm{~g} / \mathrm{l}$ picric acid-Sirius Red at $37^{\circ} \mathrm{C}$ for $1 \mathrm{~h}$, and then washed with water. The sections were mounted and viewed under a Leica DMI3000B microscope (Leica, Germany) with the use of a bright field.

2.5. Cell Preparation. Primary rat synovial fibroblasts and macrophages were obtained from additional normal rats. In brief, synovial tissues were washed for 2-3 times with phosphate-buffered saline (PBS) and then minced into pieces of $2-3 \mathrm{~mm}^{2}$ and digested in $0.1 \%$ collagenase type II (SigmaAldrich, St. Louis, MO, USA) for $30 \mathrm{~min}$. Following cell dissociation, the samples were filtered through a cell strainer. After dissociation, fibroblasts were pelleted by centrifugation at $1500 \mathrm{rpm}$ for $4 \mathrm{~min}$ and cultured in DMEM supplemented with $10 \%$ fetal bovine serum (FBS; Gibco, Thermo Fisher Scientific, Waltham, MA, USA) and antibiotics (100 U/ml penicillin, $100 \mu \mathrm{g} / \mathrm{ml}$ streptomycin; Invitrogen, CA, USA). Macrophages were obtained from the peritoneal exudates with PBS containing 2\% FBS. Cells were identified as our previous studies [15]. Passages 3-6 of the synovial fibroblasts were used for the experiments. 
TABLE 1: Nucleotide sequences of primers used for RT-PCR amplification.

\begin{tabular}{lcc}
\hline Target gene & \multicolumn{1}{c}{ Forward primer } & Reverse primer \\
\hline Caspase1 & $5^{\prime}$-TCGGAGAGTCGGAGCTGATGTT-3' & $5^{\prime}$-CTCTGGGCAGGCAGCAAATTCT-3 \\
NLRP3 & $5^{\prime}$-CCAGACCTCCAAGACCACGACT-3' & $5^{\prime}$-CCATCCGCAGCCAATGAACAGA-3' \\
ASC & $5^{\prime}$-GAGTCTGGAGCTGTGGCTACTG-3' & $5^{\prime}$-ATGAGTGCTTGCCTGTGTTGGT-3' \\
GSDMD & $5^{\prime}$-TGCTTGCCGTACTCCATTCCATC-3' & $5^{\prime}$-AGTTCTGAAGAGCCTGCCTCCA-3' \\
IL-1 $\beta$ & $5^{\prime}$-CTCATTGTGGCTGTGGAGAAG-3' & $5^{\prime}$-ACACTAGCAGGTCGTCATCAT-3' \\
IL-18 & $5^{\prime}$-ACCGCAGTAATACGGAGCAT-3' & $5^{\prime}$-TCTGGGATTCGTTGGCTGTT-3' \\
HMGB1 & $5^{\prime}$-AGTTCAAGGACCCCAATGCC-3' & $5^{\prime}$-GTCATCCGCAGCAGTGTTGTT-3' \\
TGF- $\beta$ & $5^{\prime}$-GACTCTCCACCTGCAAGACC-3' & $5^{\prime}$-GGACTGGCGAGCCTTAGTTT-3' \\
COL1A1 & $5^{\prime}$-GTACATCAGCCCAAACCCCA-3' & $5^{\prime}$-CAGGATCGGAACCTTCGCTT-3' \\
PLOD2 & $5^{\prime}$-AAGACGCTGCGATCAGAGAT-3' & $5^{\prime}$-AGTGGGGGAGTCTTTTTCCC-3' \\
TIMP1 & $5^{\prime}$-TGTGGGAAATGCCACAGGTT-3' & $5^{\prime}$-TTCCGTTCCTTAAACGGCCC-3' \\
GAPDH & $5^{\prime}$-GGCCTTCCGTGTTCCTACC-3' & $5^{\prime}$-ACTCGACACCTGCCCTCA-3' \\
\hline
\end{tabular}

2.6. Small Interfering RNA Preparation and Transfection. To inhibit the caspase1 and GSDMD expression in the macrophages, commercially available caspase1, GSDMD, and vehicle/scrambled small interfering RNA (Invitrogen, CA, USA) were used. Macrophages were transfected with siRNAs by using Lipofectamine 2000 (Invitrogen, CA, USA) according to the manufacturer's instructions. siRNA was diluted in a transfection reagent and culture medium, and the cells were incubated with $20 \mathrm{pmol}$ siRNA for $6 \mathrm{~h}$.

2.7. Cell Treatment. LPS + ATP were used to simulate the inflammatory environment of KOA and induce cell pyroptosis. Macrophages transfected with caspasel siRNA (Caspase1 siRNA group) or vehicle/scrambled siRNA (KOA group) were stimulated with LPS $(1 \mu \mathrm{g} / \mathrm{ml})$ in DMEM for $6 \mathrm{~h}$ and then challenged with ATP $(3 \mathrm{mM})$ for $1 \mathrm{~h}$. The macrophages exposed to DMEM with the same volume of PBS served as controls (Normal group).

To evaluate the relationship between pyroptosis and fibrosis, macrophages were plated into 6-well plates. The siRNA GSDMD group, the KOA group, and the Normal group were transfected with siRNA and stimulated with LPS as described above. After induction, macrophages were cultured in DMEM supplemented with 10\% FBS. Then, synovial fibroblasts precultured in a Transwell chamber were placed on a 6-well plate and cocultured with macrophages for 48 hours.

2.8. Hoechst and Propidium Iodide (PI) Staining. To evaluate pore formation in the cell membrane, Hoechst 33342 and PI (Beyotime Biotechnology, Shanghai, China) were used for staining. Briefly, macrophages were stained with Hoechst for $10 \mathrm{~min}$. After washing three times with phosphate-buffered saline, PI was added for another $10 \mathrm{~min}$. Cells were observed using a fluorescence microscope (Leica DMI3000B, Germany).
2.9. Western Blotting. Briefly, synovial tissues or cells were mixed with RIPA lysate and ground for 10-15 min. Samples were agitated on ice for $30 \mathrm{~min}$ and then the supernatant was collected. The protein levels were quantified with a BCA protein assay kit (Beyotime Biotechnology, Shanghai, China). Then, the protein samples were electrophoresed in SD-PAGE to separate protein bands. Proteins were transferred from the gel onto the PVDF membrane and blocked with $5 \%$ nonfat dry milk for $2 \mathrm{~h}$. The membrane was incubated with a primary antibody $(1: 1000$; Abcam, Cambridge, UK) overnight at $4^{\circ} \mathrm{C}$ and then with a secondary antibody (Thermo Fisher Scientific, Shanghai, China) for $2 \mathrm{~h}$. Later, bands were visualized by exposure to the ECL method and the overall gray value of the protein bands (average gray value/gray value area) was quantified; GAPDH was used as an internal marker, namely, target protein gray value/internal reference overall gray value.

2.10. Real-Time PCR. Briefly, total RNA was extracted with TRIzol and assessed by a spectrophotometer. Then, RNA from each group and $10 \mu \mathrm{l}$ PCR reaction solution were added into $10 \mu \mathrm{l}$ of the Prime Script RT Reagent Kit (Beyotime Biotechnology, Shanghai, China) for reverse transcription at conditions of $37^{\circ} \mathrm{C}(15 \mathrm{~min})$ and $85^{\circ} \mathrm{C}(5 \mathrm{~s})$. The primer was designed and synthesized by Shanghai Biotechnology Service Company in accordance with the gene sequence in the GenBank gene sequence design, together with OLIGO v6.6. Sequences are shown in Table 1. qPCR was performed using Premix Ex Taq SYBR Green PCR (TaKaRa) according to the manufacturer's instructions on an ABI PRISM 7300 (Applied Biosystems, Foster City, CA, USA). The mRNA level of individual genes was normalized to GAPDH and calculated by the $2^{-\triangle \Delta C T}$ data analysis method.

2.11. Statistical Analysis. Statistical analysis was performed using GraphPad Prism 6.0 Software (San Diego, CA, USA). Data are presented as mean \pm standard deviation (SD). Group comparisons were assessed with one-way 

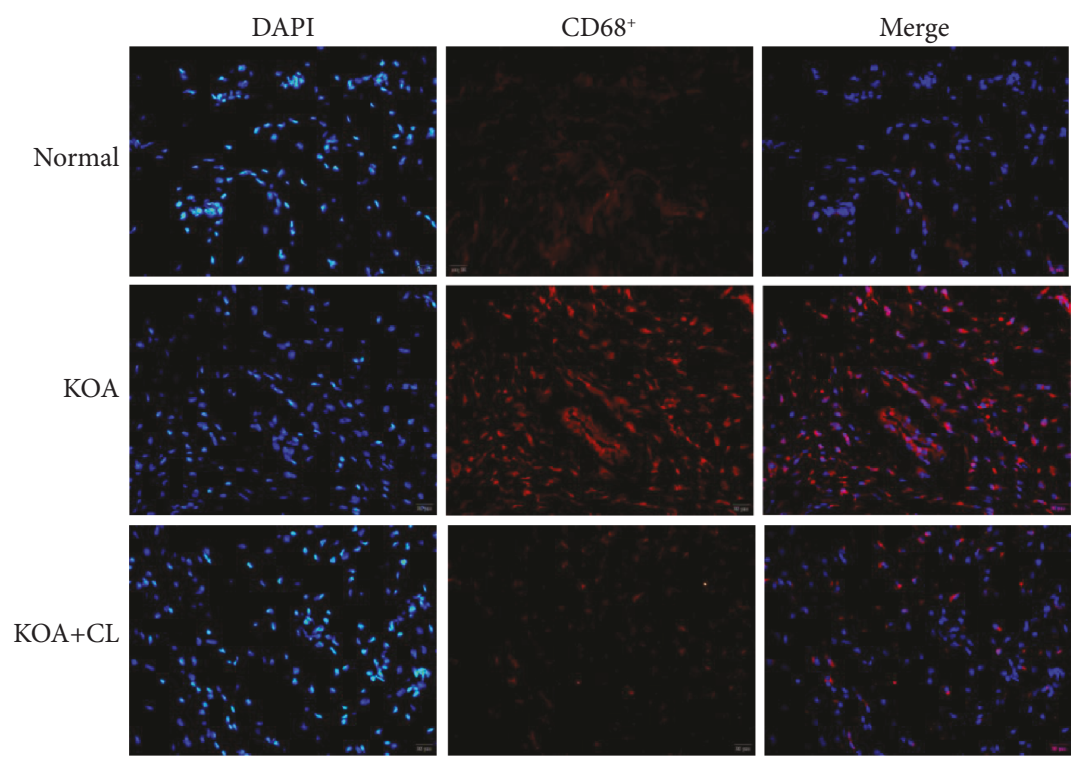

(a)

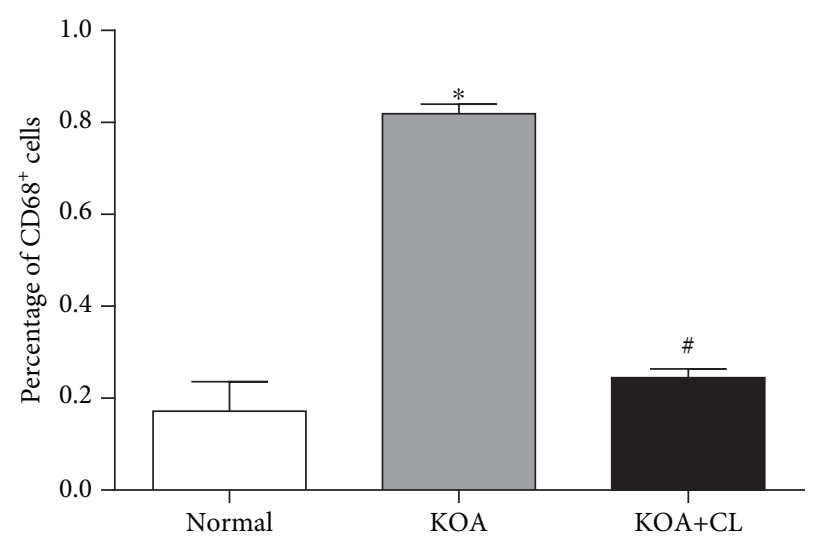

(b)

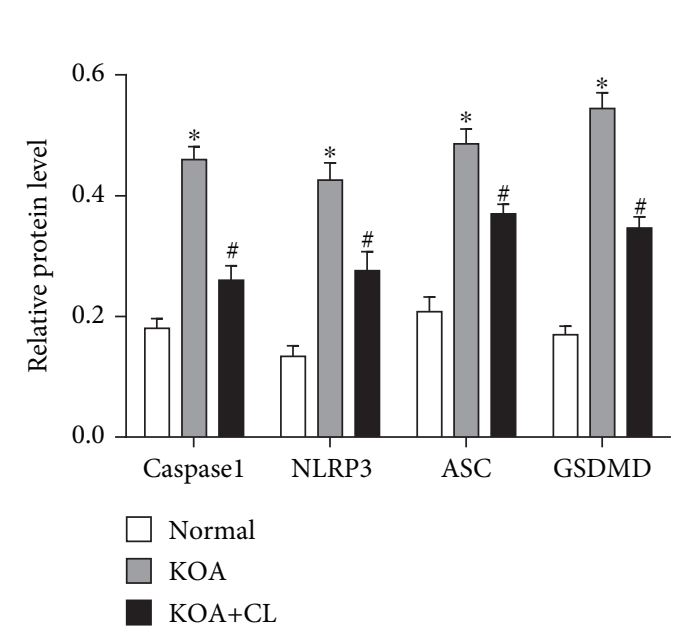

(d)

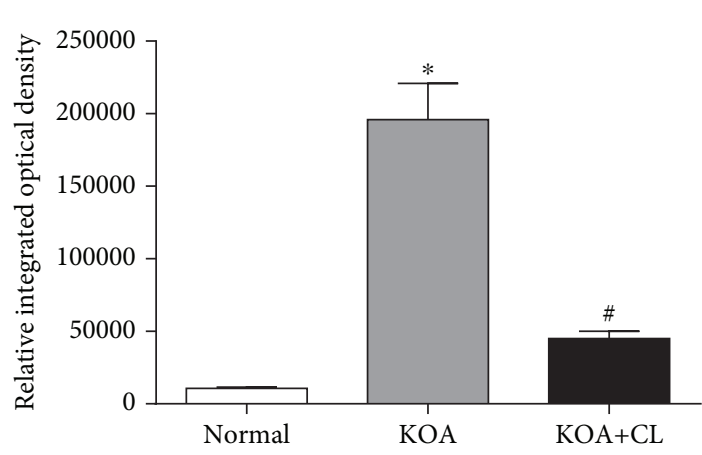

(c)

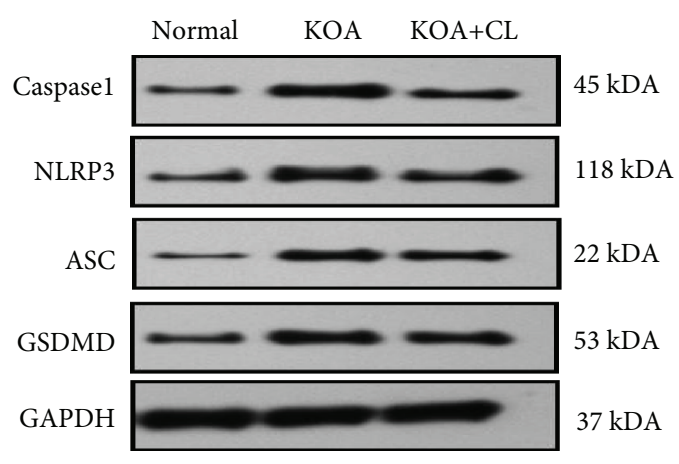

(e)

Figure 1: Continued. 


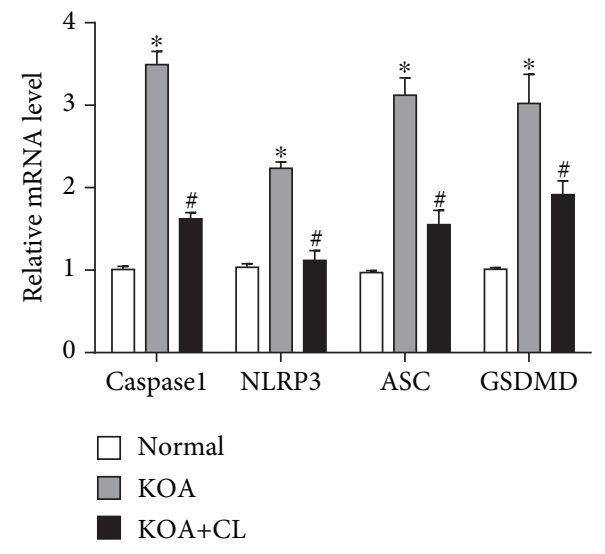

(f)

FIGURE 1: Synovial macrophage pyroptosis in KOA model rats. (a) Representative synovial tissues stained with DAPI and TRITC. Scale bar $=20 \mu \mathrm{m}(\times 100)$. (b) Percentage of $\mathrm{CD}^{+} 8^{+}$cells in each group. ${ }^{*} P<0.05$, in comparison with the Normal group. ${ }^{\#} P<0.05$, in comparison with the KOA group. (c) Relative integrated optical density (IOD) value in each group. Data were analyzed by ImageJ. (d) Protein expressions of caspase1, NLRP3, ASC, and GSDMD between groups. ${ }^{*} P<0.05$, in comparison with the Normal group. ${ }^{\#} P<0.05$, in comparison with the KOA group $(n=6)$. Western blot data are presented from three independent experiments. (e) Typical protein bands for each group. (f) mRNA level of caspase1, NLRP3, ASC, and GSDMD between groups. Trend is consistent with protein expression.

ANOVA or Student's $t$-test or two-way ANOVA with Bonferroni's post hoc test for comparison of multiple columns. A value of $P<0.05$ (two-tailed) was considered as statistically significant.

\section{Results}

3.1. Synovial Macrophage Pyroptosis in KOA Model Rats. An immunofluorescence assay (Figure 1(a)) was performed to confirm that synovial macrophages had been depleted. The KOA group resulted in a significant upregulation in the percentage of $\mathrm{CD}^{+} 8^{+}$cells (Figure 1(b)) compared with the Normal group. Meanwhile, the KOA+CL group showed a downregulation compared with the KOA group that suggests a depletion of macrophages in the $\mathrm{KOA}+\mathrm{CL}$ group. Comparison of the relative integrated optical density (IOD) value in each group was consistent with the variation tendency of $\mathrm{CD}^{+} 8^{+}$cells (Figure 1(c)). Subsequently, we analyzed both gene and protein expressions of caspase1, NLRP3, and ASC, which constitute the NLRP3 inflammasome. Our data showed that the KOA group resulted in a significant upregulation (Figures 1(d)-1(f)) compared with the Normal group, and the $\mathrm{KOA}+\mathrm{CL}$ group showed a downregulation compared with the KOA group. In addition, the GSDMD level showed the same trend.

3.2. Inhibition of Synovial Macrophage Pyroptosis May Alleviate Synovitis in KOA Rats. In order to evaluate synovitis, hematoxylin and eosin staining was performed. Compared with the KOA group, the KOA+AYC group showed an orderly arranged lining of synovial cells, loose connective tissue, and less inflammatory cell infiltration (Figure 2(a)). In addition, both mRNA and protein expressions of IL- $1 \beta$, IL18, and HMGB1 were measured (Figures 2(b)-2(d)). We found that there was a significant decrease in the expression of these proinflammatory cytokines in the KOA+AYC group.
3.3. Inhibition of Synovial Macrophage Pyroptosis May Alleviate Fibrosis in KOA Rats. To quantify the degree of knee swelling in rats, transverse diameters of the right knees were measured (Figure 3(a)). On Day 14, the knee joint diameter of the KOA group was significantly larger than that of the Normal group, and on Day 28, the knee joint diameter of the $\mathrm{KOA}+\mathrm{AYC}$ group was significantly smaller than that of the KOA group. To observe the fibrosis of synovial tissue, we carried out Sirius Red staining. Compared with the Normal group, the KOA group showed markedly increased collagen deposition. This change was alleviated in the KOA+AYC group (Figure 3(b)). Semiquantification of Sirius Red staining (Figure 3(c)) was evaluated using the average integral optical density (AIOD). The KOA+AYC group showed a significant downregulation compared with the KOA group. Furthermore, we analyzed mRNA and protein expressions of TGF- $\beta$, PLOD2, COL1A1, and TIMP1 (Figures $4(\mathrm{~d})-4(\mathrm{f}))$. We found that there was a significant decrease in the level of these profibrotic substances in the KOA+AYC group.

3.4. Pyroptosis Is Induced in LPS+ATP-Treated Macrophages. An LPS+ATP-induced model in macrophages was established mimicking the inflammatory environment of KOA and inducing macrophage pyroptosis. The silencing effect of the caspase1 siRNA and GSDMD siRNA was confirmed by PCR (Figure 4(a)). Hoechst and PI staining showed that the number of pyroptotic cells was increased by LPS+ATP treatment, and this trend can be reversed by caspase1 siRNA (Figure 4(b)). Besides, the protein expressions of NLRP3, ASC, GSDMD, and cleavage-GSDMD (GSDMD-N) in the KOA group were higher than those in the Normal group, and the Caspase1 siRNA group showed a significant decrease of these key pyroptosis-related substances compared with the KOA group (Figures 4(c) and 4(d)). 

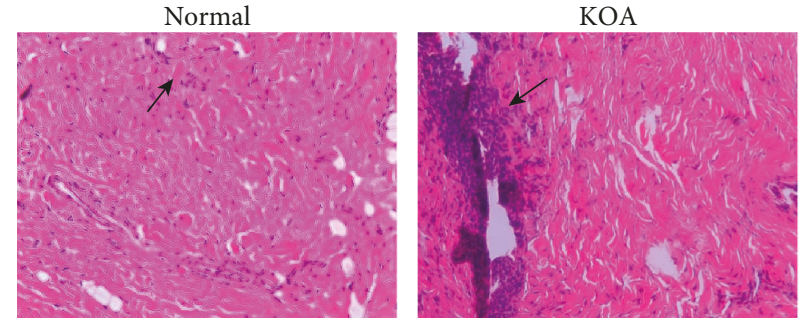

(a)
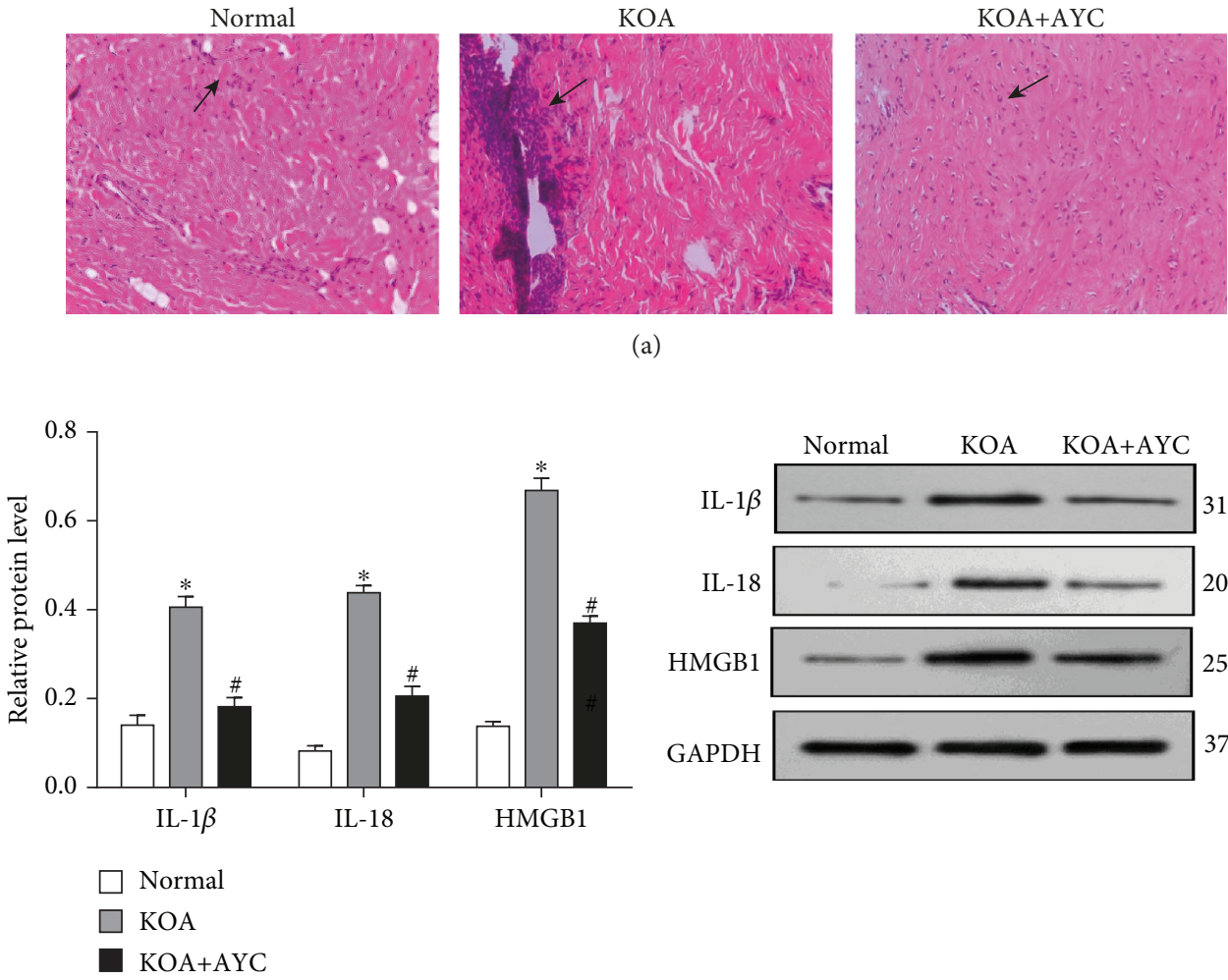

(b)

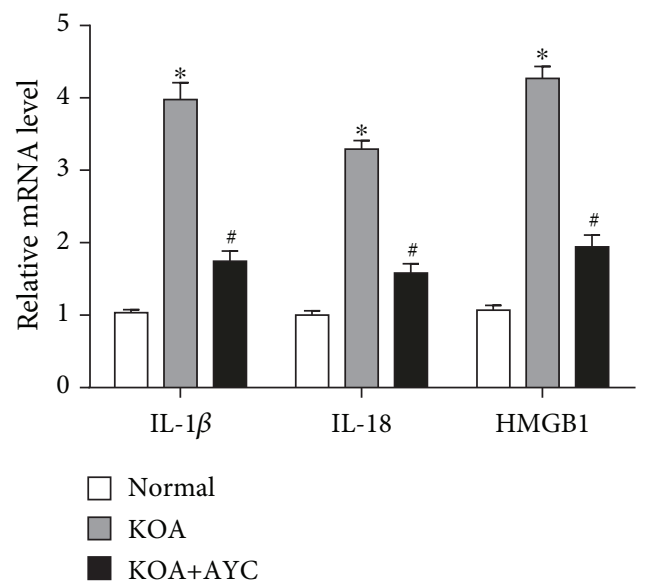

(d)

FIGURE 2: Inhibition of synovial macrophage pyroptosis may alleviate synovitis in KOA rats. (a) Representative synovial tissues of each group stained with HE staining $(200 \times)$. Arrow: inflammatory cell infiltration. (b) Protein expressions of IL-1 $\beta$, IL-18, and HMGB1 between groups. ${ }^{*} P<0.05$, in comparison with the Normal group. ${ }^{\#} P<0.05$, in comparison with the KOA group $(n=6)$. (c) Typical protein bands for each group. (d) mRNA levels of IL-1 $\beta$, IL-18, and HMGB1 between groups. The trend is consistent with protein expression.

3.5. Macrophage Pyroptosis Upregulates the Expression of Fibrosis Markers Cocultured with Synovial Fibroblasts. After coculture for 48 hours, both mRNA and protein expressions of TGF- $\beta$, PLOD2, COL1A1, and TIMP1 in synovial fibroblasts were detected. These fibrosis markers showed a significant upregulation in the KOA group compared with the Normal group, and a downregulation compared with the siRNA GSDMD group (Figures 5(a)-5(c)).

\section{Discussions}

The current study indicates that synovial macrophage pyroptosis participates in the pathologic process of KOA. Inhibition of synovial macrophage pyroptosis alleviates synovitis and fibrosis in KOA. ACLT surgery was performed to construct KOA model rats. ACLT is modified from the Hulth method and widely used for modeling 


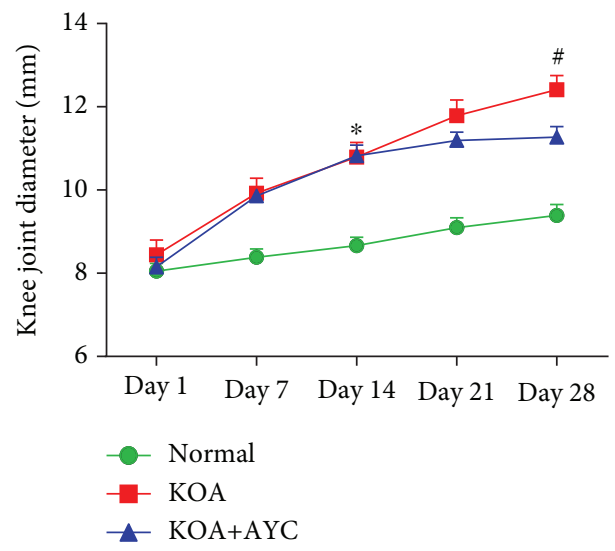

(a)

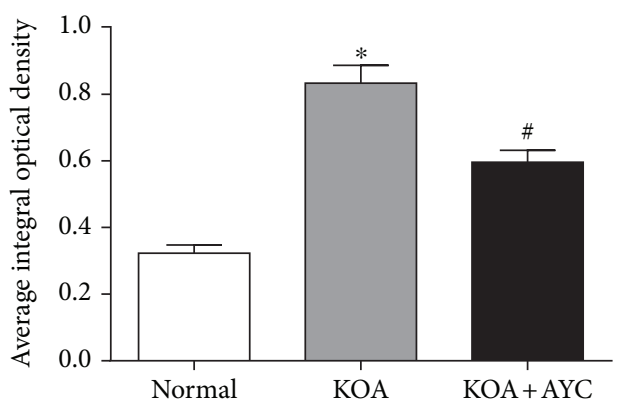

(c)

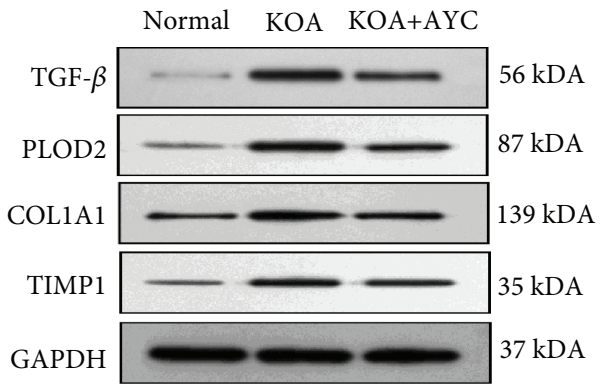

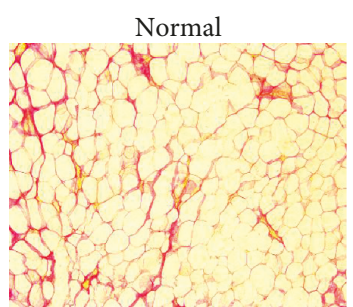

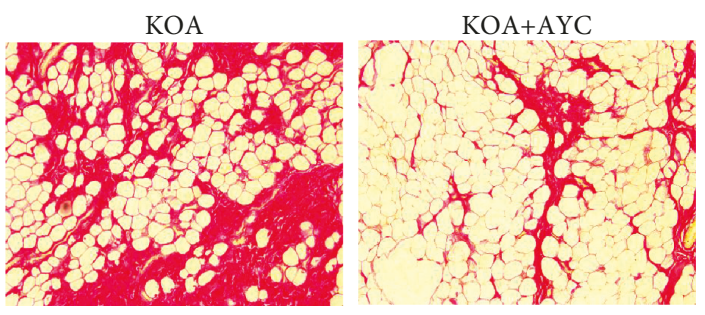

(b)

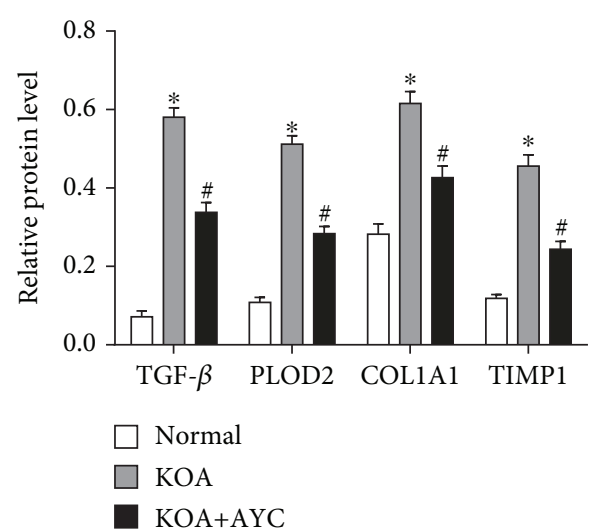

(d)

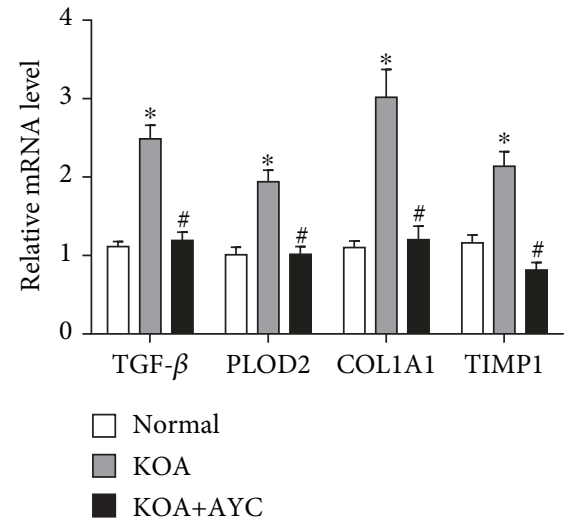

(f)

FIGURE 3: Inhibition of synovial macrophage pyroptosis may alleviate fibrosis in KOA rats. (a) The severity of synovial fibrosis evaluated by the transverse diameters of the right knees. ${ }^{*} P<0.05$ vs. the Normal group; ${ }^{*} P<0.05$ vs. the KOA+AYC group. (b) Collagen deposition was revealed through Sirius Red staining $(200 \times)$. (c) Semiquantification of Sirius Red staining was evaluated using the average integral optical density (AIOD). The results were expressed as the mean $\pm \mathrm{SD}(n=6) .{ }^{*} P<0.05$, in comparison with the Normal group. ${ }^{\#} P<0.05$, in comparison with the KOA group. Data were analyzed by ImageJ. (d) Comparison of TGF- $\beta$, PLOD2, COL1A1, and TIMP1 protein expressions between groups. ${ }^{*} P<0.05$, in comparison with the Normal group. ${ }^{\#} P<0.05$, in comparison with the KOA group $(n=6)$. (e) Typical protein bands for each group. (f) The mRNA levels of TGF- $\beta$, PLOD2, COL1A1, and TIMP1 between groups.

KOA. ACLT results in joint instability and thus induces cartilage degeneration, subchondral bone sclerosis, and osteophyte formation, which mimics the pathological changes observed in human OA [22]. This method has less trauma and physiological structure changes than the Hulth method and develops less rapidly compared with MIA method [23]. Therefore, it is more suitable for this study. 


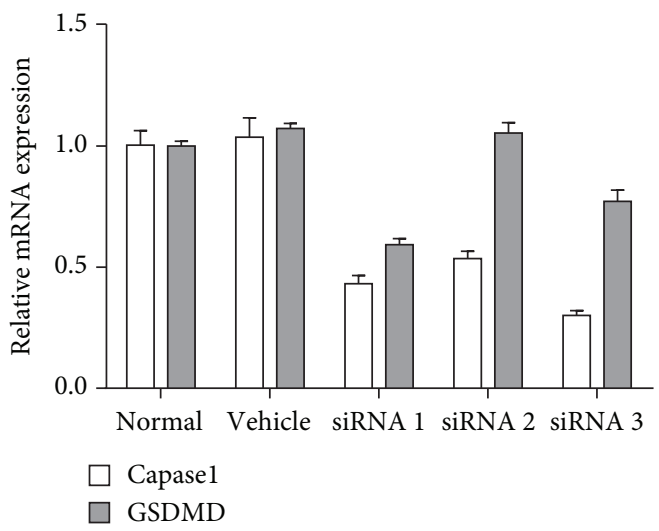

(a)
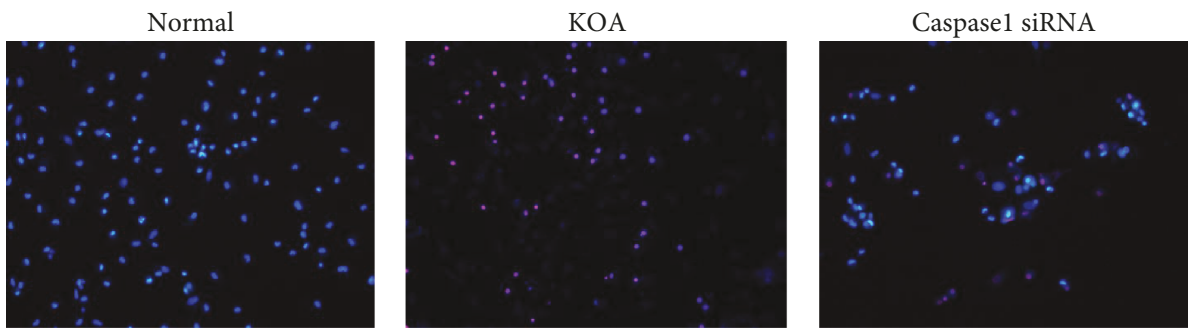

(b)

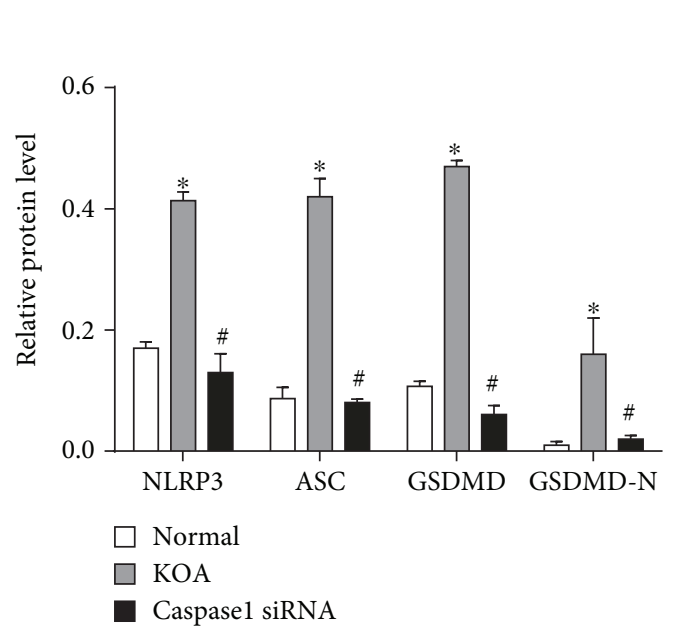

(c)

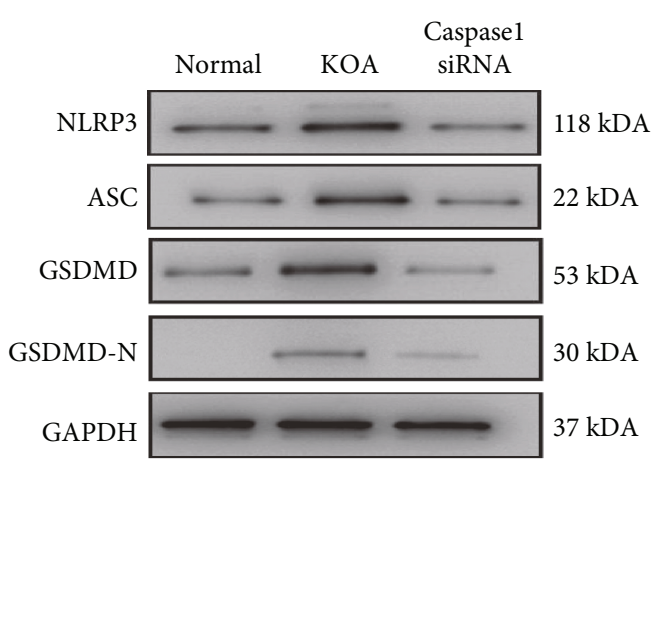

(d)

FIGURE 4: Pyroptosis is induced in LPS+ATP-treated macrophages. (a) The silencing effects of the caspase1 siRNA and GSDMD siRNA were both confirmed by PCR. (b) Pyroptotic cells observed by Hoechst and PI staining (200×). (c) Comparison of NLRP3, ASC, GSDMD, and GSDMD-N protein expressions between groups. ${ }^{*} P<0.05$, in comparison with the Normal group. ${ }^{\#} P<0.05$, in comparison with the KOA group. (d) Typical protein bands for each group.

We revealed that pyroptosis occurs in synovial macrophages by comparing synovial tissue with or without the depletion of synovial macrophages. We also conducted a series of quantitative studies to demonstrate that synovial macrophage pyroptosis is related to synovitis and fibrosis in vivo. In addition, we found the high expression of fibrosis markers induced by pyroptosis through macrophages and synovial fibroblasts cocultured in vitro. Since the symptoms of KOA are directly related to the severity of synovitis and fibrosis, the findings of this paper may suggest a novel role for synovial macrophages in the pathogenesis of KOA.
Macrophages play a crucial role in the progression of KOA and synovial macrophage activity, and their mediators have been regarded as potential therapeutic targets in OA. While exposed to inflammatory conditions, macrophages can become activated and may acquire a phenotype, ranging from proinflammatory (M1) to anti-inflammatory (M2) phenotypes [24]. Utomo et al. proved that macrophage phenotype modulation can be used to guide joint inflammation. Furthermore, the team also proved that cartilage inflammation and degeneration could be enhanced by M1 macrophages via the upregulation of IL-1 $\beta$, IL-6, MMP-13, and 


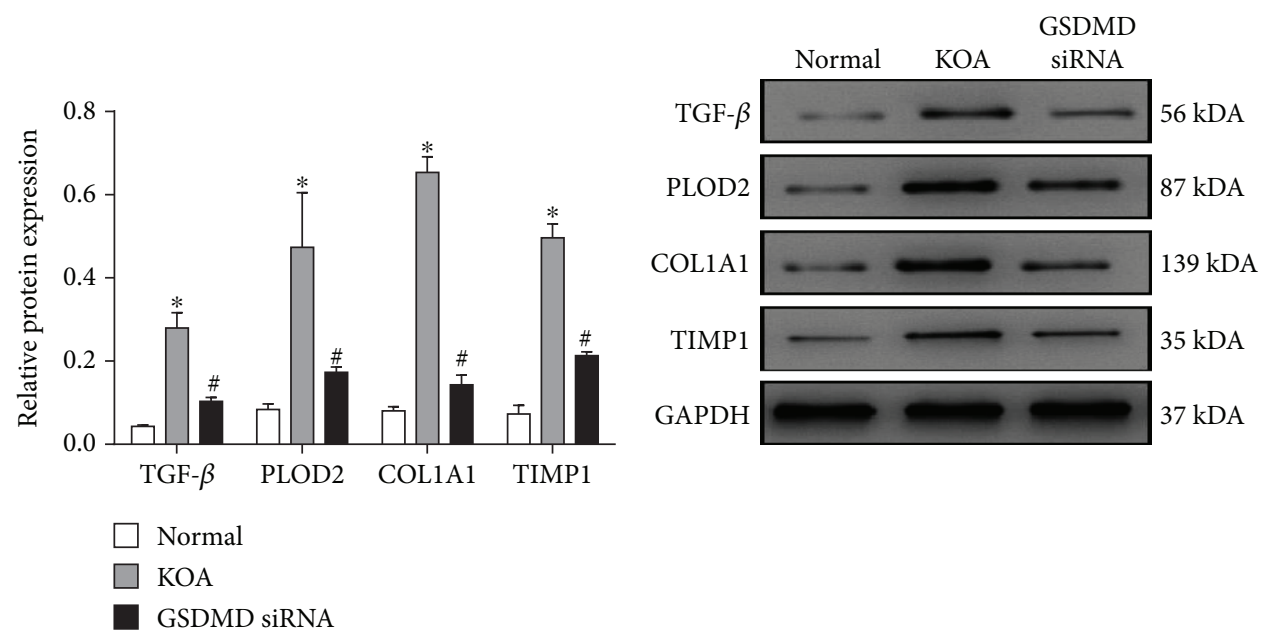

(a)

(b)

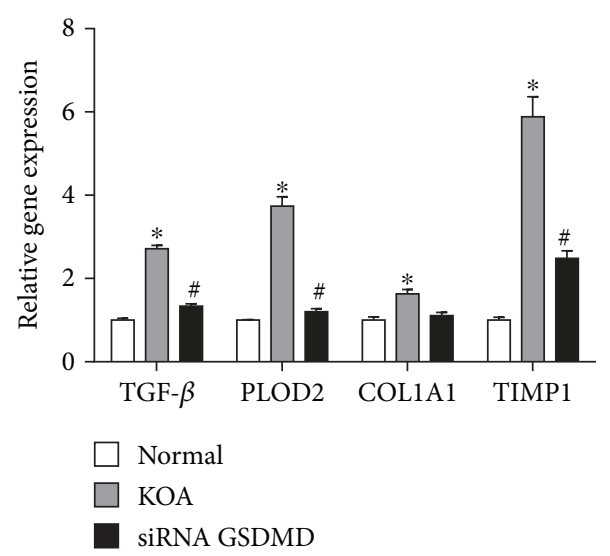

(c)

Figure 5: Macrophage pyroptosis upregulates the expression of fibrosis markers cocultured with synovial fibroblasts. (a) Comparison of TGF- $\beta$, PLOD2, COL1A1, and TIMP1 protein expressions between groups. ${ }^{*} P<0.05$, in comparison with the Normal group. ${ }^{\#} P<0.05$, in comparison with the KOA group. (b) Typical protein bands for each group. (c) Relative mRNA expression of TGF- $\beta$, COL1A1, PLOD2, and TIMP1 in synovial fibroblasts in each group. ${ }^{*} P<0.05$, in comparison with the Normal group. ${ }^{\#} P<0.05$, in comparison with the KOA group.

ADAMTS-5 [25, 26]. Increasing evidence has shown that macrophage pyroptosis in different tissues such as liver, renal, and airway tissues participates in chronic aseptic inflammation and can be related to tissue fibrosis. It is also widely recognized that the cleavage of GSDMD plays a crucial role in pyroptosis [16-18]. In the canonical pathway, pyroptosis may require a two-step mechanism. At the first stage, proinflammatory mediators pro-IL- $1 \beta$ and pro-IL-18, caspase1, and NLRP family members are transcriptionally generated. The second stage is activation, including the NLRP inflammasomes assembling and caspase1 activating. Activated caspase1 further causes pro-IL-1 $\beta$ and pro-IL-18 to mature and induces pyroptosis depending on the cleavage of GSDMD [11]. Unlike the canonical pathway, caspase1 can be activated by directly sensing intracellular LPS and by promoting pyroptosis through GSDMD cleavage; this is the socalled noncanonical pathway pyroptosis [27]. Pyroptosis causes rapid plasma membrane rupture, resulting in the release of intracellular proinflammatory mediators IL- $1 \beta$, IL-18, and HMGB1 [28]. In this paper, both mRNA and pro- tein expressions of caspase1, NLRP3, ASC, and GSDMD in the KOA synovial tissue resulted in a significant upregulation compared with the Normal group. Meanwhile, by the depletion of macrophages with Clodronate Liposomes, the KOA $+\mathrm{CL}$ group showed a downregulation of these pyroptosisrelated proteins compared with the KOA group. In addition, LPS+ATP-treated macrophages showed an increase in the number of pyroptotic cells and the protein expression of NLRP3, ASC, GSDMD, and GSDMD-N compared with the Normal group; this trend could be reversed by siRNA caspase1. Evidence above all suggests that pyroptosis occurs in synovial macrophages of KOA.

IL- $1 \beta$ and IL- 18 are not only the downstream products of pyroptosis but are also important promoters of inflammation. IL-1 $\beta$, as well as HMGB1, acts as a "gate keeper" of inflammation and directly contributes to synovial inflammation. In order to observe the relationship between macrophage pyroptosis and synovitis, HE staining was performed. Caspase1-inhibitor-treated rats (KOA+AYC group) showed a more orderly arranged lining of synovial cells and less 
inflammatory cell infiltration. In addition, both mRNA and protein expressions of IL-1 $\beta$, IL-18, and HMGB1 were decreased in the $\mathrm{KOA}+\mathrm{AYC}$ group. They are not only indicators for synovitis but are also downstream evidence for pyroptosis. Therefore, we speculate that synovial macrophage pyroptosis could promote synovitis, further contributing to KOA progression.

The response to TGF- $\beta$ is a key event in the onset of synovial fibrosis [29]. Xue et al. have proven that smad4 silencing can suppress chronic inflammation and fibrosis in joint tissues by inhibiting the TGF- $\beta /$ Smad pathway and can play a positive role in the prevention and treatment of joint stiffness [30]. In addition, gene expressions of PLOD2, COL1A1, and TIMP1 were upregulated both in OA fibroblasts stimulated with TGF- $\beta$ and in mice with TGF- $\beta$ induced fibrosis [31]. Accordingly, we first measured the transverse diameters of the right knees to quantify the degree of knee swelling in rats and performed Sirius Red staining to observe collagen deposition. Then, we measured both mRNA and protein expressions of TGF- $\beta$, PLOD2, COL1A1, and TIMP1 to evaluate fibrosis in synovium. The KOA+AYC group showed a downregulation of all these profibrotic substances compared with the KOA group. Subsequently, as synovial fibroblasts are the main effector cells of synovial fibrosis, we established a coculture model of macrophages and synovial fibroblasts. After coculture for 48 hours, both mRNA and protein expressions of TGF- $\beta$, PLOD2, COL1A1, and TIMP1 in synovial fibroblasts showed a significant upregulation in the KOA group compared with the Normal group and a downregulation compared with the siRNA GSDMD group. Therefore, we speculate that the inhibition of synovial macrophage pyroptosis could alleviate fibrosis.

The current study still has a few limitations. First, due to the limitation of experimental conditions, we failed to extract macrophages from synovial tissue directly. We have tried the method of sorting magnetic beads, but the number of synovial macrophages obtained is really small. Fortunately, the extraction of macrophages from the peritoneal exudates as an alternative has been supported by many researchers [32-34]. As in clinical studies, macrophages in the peripheral blood of KOA patients are often used as substitutes for synovial macrophages. Secondly, the sample size of experimental animals would be bigger if possible. Future studies will focus on the exploration of medicine monomers which could inhibit pyroptosis in order to alleviate synovitis and fibrosis in KOA.

\section{Conclusion}

In summary, although a few limitations exist, this study still has demonstrated that the pyroptosis of synovial macrophages occurs in the development of KOA rats, which partly explains the pathogenesis of KOA. In addition, the study also indicates that synovial macrophages could be an effective target for the treatment of synovitis and fibrosis in knee osteoarthritis. The inhibition of synovial macrophage pyroptosis alleviates synovitis and fibrosis in $\mathrm{KOA}$; therefore, it provides a new target for the clinical treatment of KOA.

\author{
Abbreviations \\ KOA: Knee osteoarthritis \\ FLS: $\quad$ Fibroblast-like synoviocytes \\ NLRP: Nod-like receptor protein \\ ASC: $\quad$ Apoptosis-associated speck-like protein with a \\ caspase-recruitment domain \\ GSDMD: Gasdermin D \\ LPS: $\quad$ Lipopolysaccharide \\ ATP: $\quad$ Adenosine triphosphate \\ siRNAs: Small interfering RNAs \\ HIF-1 $\alpha$ : Hypoxia-inducible factors- $1 \alpha$ \\ TGF- $\beta$ : $\quad$ Transforming growth factor- $\beta$ \\ PLOD2: $\quad$ Procollagen-lysine, 2-oxoglutarate 5-dioxygenase 2 \\ COL1A1: Collagen type I $\alpha 1$ chain \\ TIMP1: Tissue inhibitor of metalloproteinase 1.
}

\section{Data Availability}

The data used to support the findings of this study are available from the corresponding author upon request.

\section{Conflicts of Interest}

All authors declare that there are no actual or potential conflicts of interest, including any financial, personal, or other relationships with other people or organizations that could inappropriately influence, or be perceived to influence, our work.

\section{Acknowledgments}

The current work was supported by the National Natural Science Foundation of China (Nos. 81573993 and 81774334), the Leading Talents of Traditional Chinese Medicine Project (SLJ0207), and the Cultivation Project of Affiliated Hospital of Nanjing University of Chinese Medicine (Y17001).

\section{References}

[1] T. Hügle and J. Geurts, "What drives osteoarthritis?-Synovial versus subchondral bone pathology," Rheumatology, vol. 56, no. 9, pp. 1461-1471, 2017.

[2] S. Glyn-Jones, A. J. R. Palmer, R. Agricola et al., "Osteoarthritis," The Lancet, vol. 386, no. 9991, pp. 376-387, 2015.

[3] M. D. Smith, "The normal synovium," The Open Rheumatology Journal, vol. 5, no. 1, pp. 100-106, 2011.

[4] E. B. P. Lopes, A. Filiberti, S. A. Husain, and M. B. Humphrey, "Immune contributions to osteoarthritis," Current Osteoporosis Reports, vol. 15, no. 6, pp. 593-600, 2017.

[5] V. B. Kraus, G. McDaniel, J. L. Huebner et al., "Direct in vivo evidence of activated macrophages in human osteoarthritis," Osteoarthritis and Cartilage, vol. 24, no. 9, pp. 1613-1621, 2016.

[6] D. F. G. Remst, E. N. Blaney Davidson, E. L. Vitters et al., "Osteoarthritis-related fibrosis is associated with both elevated pyridinoline cross-link formation and lysyl hydroxylase $2 \mathrm{~b}$ expression," Osteoarthritis and Cartilage, vol. 21, no. 1, pp. 157-164, 2013. 
[7] K. K. Kim, D. Sheppard, and H. A. Chapman, “TGF- $\beta 1$ signaling and tissue fibrosis," Cold Spring Harbor Perspectives in Biology, vol. 10, no. 4, 2018.

[8] A. H. Györfi, A. E. Matei, and J. H. W. Distler, "Targeting TGF- $\beta$ signaling for the treatment of fibrosis," Matrix Biology, vol. 68-69, pp. 8-27, 2018.

[9] L. Vande Walle and M. Lamkanfi, "Pyroptosis," Current Biology, vol. 26, no. 13, pp. R568-R572, 2016.

[10] A. Mathur, J. A. Hayward, and S. M. Man, "Molecular mechanisms of inflammasome signaling," Journal of Leukocyte Biology, vol. 103, no. 2, pp. 233-257, 2018.

[11] J. Shi, Y. Zhao, K. Wang et al., "Cleavage of GSDMD by inflammatory caspases determines pyroptotic cell death," Nature, vol. 526, no. 7575, pp. 660-665, 2015.

[12] J. Shi, W. Gao, and F. Shao, "Pyroptosis: gasdermin-mediated programmed necrotic cell death," Trends in Biochemical Sciences, vol. 42, no. 4, pp. 245-254, 2017.

[13] L. R. Zhao, R. L. Xing, P. M. Wang et al., "NLRP1 and NLRP3 inflammasomes mediate LPS/ATP-induced pyroptosis in knee osteoarthritis," Molecular Medicine Reports, vol. 17, no. 4, pp. 5463-5469, 2018.

[14] L. Zhang, L. Zhang, Z. Huang et al., "Increased HIF-1 $\alpha$ in knee osteoarthritis aggravate synovial fibrosis via fibroblast-like synoviocyte pyroptosis," Oxidative Medicine and Cellular Longevity, vol. 2019, Article ID 6326517, 11 pages, 2019.

[15] T. Bergsbaken, S. L. Fink, and B. T. Cookson, "Pyroptosis: host cell death and inflammation," Nature Reviews Microbiology, vol. 7, no. 2, pp. 99-109, 2009.

[16] S. Wang, Y. H. Yuan, N. H. Chen, and H. B. Wang, "The mechanisms of NLRP3 inflammasome/pyroptosis activation and their role in Parkinson's disease," International Immunopharmacology, vol. 67, pp. 458-464, 2019.

[17] A. Wree, A. Eguchi, M. D. McGeough et al., "NLRP3 inflammasome activation results in hepatocyte pyroptosis, liver inflammation, and fibrosis in mice," Hepatology, vol. 59, no. 3, pp. 898-910, 2014.

[18] G. Lorenz, M. N. Darisipudi, and H. J. Anders, "Canonical and non-canonical effects of the NLRP3 inflammasome in kidney inflammation and fibrosis," Nephrology Dialysis Transplantation, vol. 29, no. 1, pp. 41-48, 2014.

[19] S. S. Glasson, T. J. Blanchet, and E. A. Morris, "The surgical destabilization of the medial meniscus (DMM) model of osteoarthritis in the 129/SvEv mouse," Osteoarthritis and Cartilage, vol. 15, no. 9, pp. 1061-1069, 2007.

[20] Y. Sakurai, M. Fujita, S. Kawasaki et al., "Contribution of synovial macrophages to rat advanced osteoarthritis pain resistant to cyclooxygenase inhibitors," Pain, vol. 160, no. 4, pp. 895907, 2019.

[21] H. Strobl, C. Scheinecker, B. Csmarits, O. Majdic, and W. Knapp, "Flow cytometric analysis of intracellular CD6 8 molecule expression in normal and malignant haemopoiesis," British Journal of Haematology, vol. 90, no. 4, pp. 774-782, 1995.

[22] S. V. Naveen, R. E. Ahmad, W. J. Hui et al., "Histology, glycosaminoglycan level and cartilage stiffness in monoiodoacetate-induced osteoarthritis: comparative analysis with anterior cruciate ligament transection in rat model and human osteoarthritis," International Journal of Medical Sciences, vol. 11, no. 1, pp. 97-105, 2014.

[23] J. E. Kim, D. H. Song, S. H. Kim, Y. Jung, and S. J. Kim, "Development and characterization of various osteoarthritis models for tissue engineering," PLoS One, vol. 13, no. 3, article e0194288, 2018.

[24] B. Liu, M. Zhang, J. Zhao, M. Zheng, and H. Yang, "Imbalance of M1/M2 macrophages is linked to severity level of knee osteoarthritis," Experimental and Therapeutic Medicine, vol. 16, no. 6, pp. 5009-5014, 2018.

[25] L. Utomo, G. J. V. M. van Osch, Y. Bayon, J. A. N. Verhaar, and Y. M. Bastiaansen-Jenniskens, "Guiding synovial inflammation by macrophage phenotype modulation: an in vitro study towards a therapy for osteoarthritis," Osteoarthritis and Cartilage, vol. 24, no. 9, pp. 1629-1638, 2016.

[26] L. Utomo, Y. M. Bastiaansen-Jenniskens, J. A. N. Verhaar, and G. J. V. M. van Osch, "Cartilage inflammation and degeneration is enhanced by pro-inflammatory (M1) macrophages in vitro, but not inhibited directly by anti-inflammatory (M2) macrophages," Osteoarthritis and Cartilage, vol. 24, no. 12, pp. 2162-2170, 2016.

[27] N. Kayagaki, I. B. Stowe, B. L. Lee et al., "Caspase-11 cleaves gasdermin D for non-canonical inflammasome signalling," Nature, vol. 526, no. 7575, pp. 666-671, 2015.

[28] L. Hou, Z. Yang, Z. Wang et al., "NLRP3/ASC-mediated alveolar macrophage pyroptosis enhances HMGB1 secretion in acute lung injury induced by cardiopulmonary bypass," Laboratory Investigation, vol. 98, no. 8, pp. 1052-1064, 2018.

[29] X. M. Meng, D. J. Nikolic-Paterson, and H. Y. Lan, "TGF- $\beta$ : the master regulator of fibrosis," Nature Reviews Nephrology, vol. 12, no. 6, pp. 325-338, 2016.

[30] M. Xue, S. Gong, J. Dai, G. Chen, and J. Y. Hu, "The treatment of fibrosis of joint synovium and frozen shoulder by Smad4 gene silencing in rats," PLoS One, vol. 11, no. 6, article e0158093, 2016.

[31] D. F. G. Remst, A. B. Blom, E. L. Vitters et al., "Gene expression analysis of murine and human osteoarthritis synovium reveals elevation of transforming growth factor $\beta$-responsive genes in osteoarthritis-related fibrosis," Arthritis \& Rhematology, vol. 66, no. 3, pp. 647-656, 2014.

[32] H. Zhang, C. Lin, C. Zeng et al., "Synovial macrophage M1 polarisation exacerbates experimental osteoarthritis partially through R-spondin-2," Annals of the Rheumatic Diseases, vol. 77, no. 10, pp. 1524-1534, 2018.

[33] Y. Pei, F. Cui, X. du et al., "Antioxidative nanofullerol inhibits macrophage activation and development of osteoarthritis in rats," International Journal of Nanomedicine, vol. 14, pp. 4145-4155, 2019.

[34] Z. Ni, L. Kuang, H. Chen et al., "The exosome-like vesicles from osteoarthritic chondrocyte enhanced mature IL- $1 \beta$ production of macrophages and aggravated synovitis in osteoarthritis," Cell Death \& Disease, vol. 10, no. 7, p. 522, 2019. 


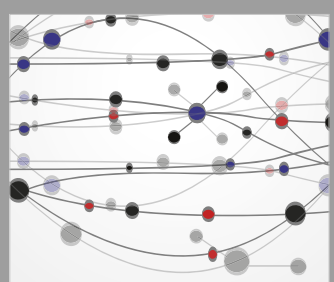

The Scientific World Journal
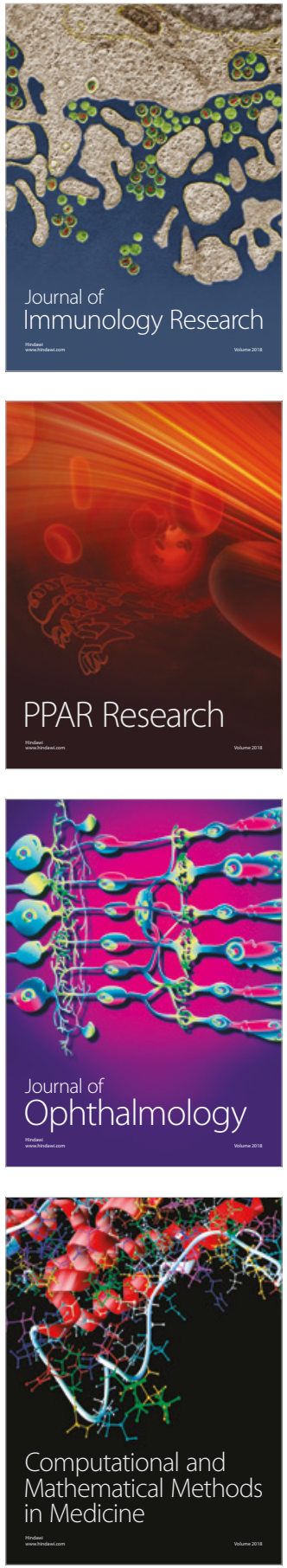

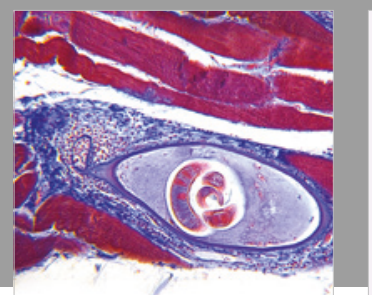

Gastroenterology Research and Practice

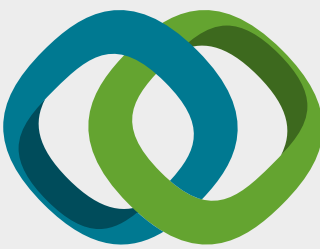

\section{Hindawi}

Submit your manuscripts at

www.hindawi.com
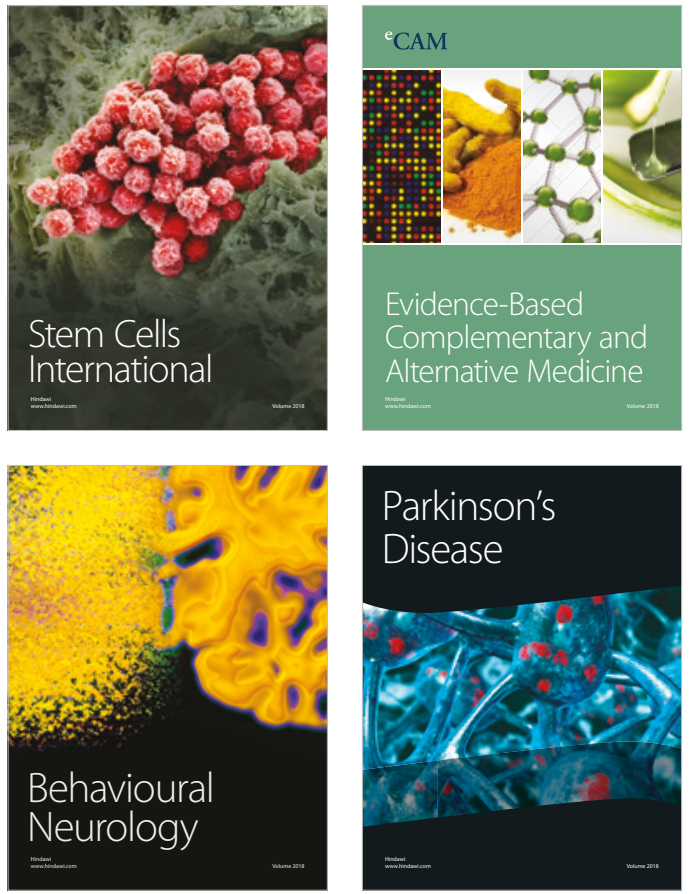

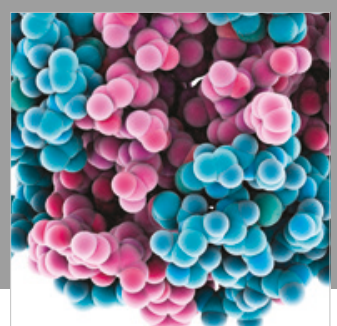

ournal of

Diabetes Research

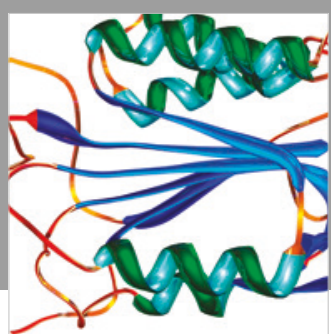

Disease Markers
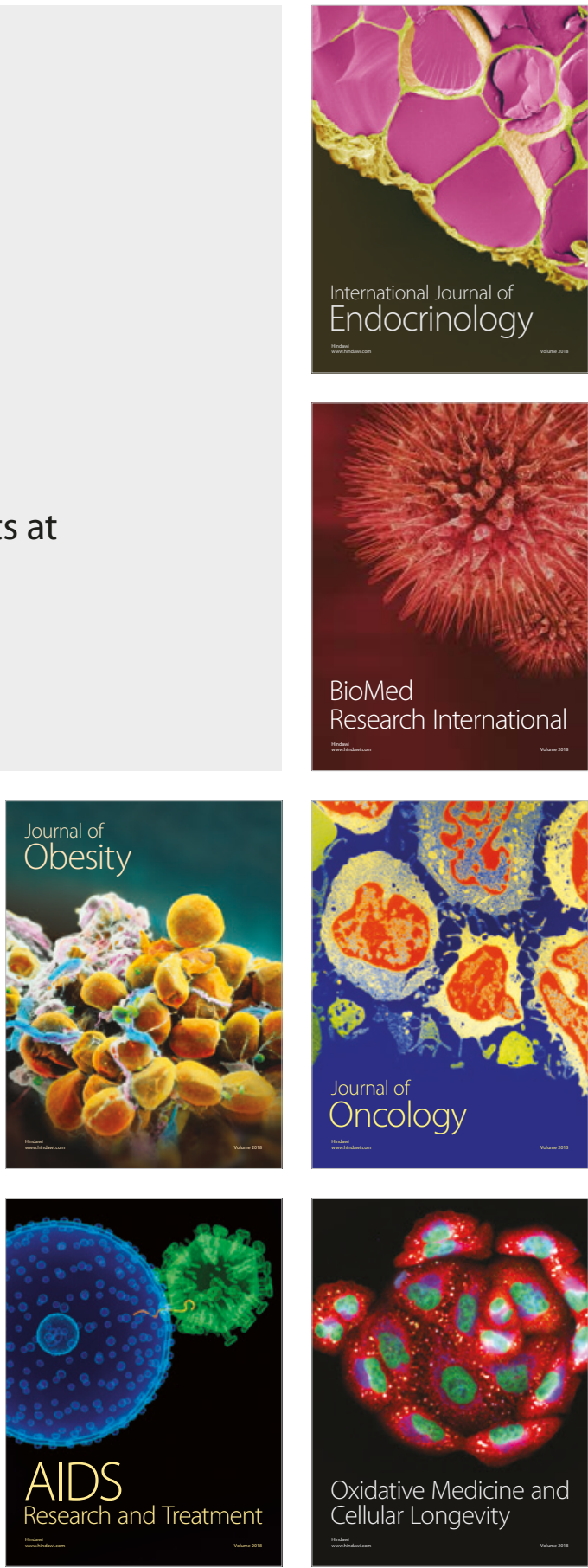